\title{
TERRITORIO, TRANSFORMACIÓN PRODUCTIVA, EQUIDAD Y REDES ${ }^{12}$
}

\section{TERRITORY, ECONOMIC CHANGE, EQUITY AND NETWORKS}

\author{
Simón Sánchez-Moral \\ Universidad Complutense de Madrid \\ simon.sanchez@ghis.ucm.es
}

Recibido: septiembre, 2014.

Versión final aceptada: diciembre, 2014.

PALABRAS CLAVE: Crisis económica, crecimiento inteligente, regiones españolas.

KEY WORDS: Economic crisis, smart growth, Spanish regions.

\section{RESUMEN}

La crisis económica ha puesto de manifiesto los desequilibrios de la economía española. El "crecimiento inteligente" de la Comisión Europea puede marcar el camino para lograr un cambio de modelo productivo, impulsando una economía basada en el conocimiento y la innovación, con mayores retornos desde la perspectiva de la cohesión social y territorial. En este trabajo se analiza el comportamiento de las estructuras sectoriales y de los sistemas regionales de innovación durante la crisis, según propone la metodología de las estrategias de especialización inteligente. Una primera muestra de estas estrategias en regiones españolas es comentada desde la perspectiva particular de las visiones y prioridades definidas por los propios actores del territorio.

\section{ABSTRACT}

The economic crisis has highlighted the imbalances of the Spanish economy. The "smart growth" of European Commission could represent a roadmap for achieving a change of the production model, fostering knowledge and innovation-based economy, with greater returns from the perspective of social and territorial cohesion. This work analyzes the transformation of sectoral structures and regional innovation systems during the crisis, as proposed by the methodology of smart specialization strategies. An initial sample of these strategies from Spanish regions is discussed specifically from the point of view of visions and priorities defined by the actors of territory.

\footnotetext{
${ }^{1}$ Este trabajo está dedicado a la memoria de Julio Cesar Ondategui (1957-2013).

2 Simón Sánchez Moral es investigador del subprograma "Ramón y Cajal" del Ministerio de Ciencia e Innovación. Este texto forma parte de los proyectos del Plan Nacional de I+D+i. "Efectos socioterritoriales de la crisis económica en las áreas urbanas de España: políticas públicas y estrategias de resiliencia" (CS02012-36170) y "Lugares, imaginarios y movilidades turísticas en tiempos de crisis" (CSO2011-26527). El autor quiere agradecer al profesor Emilio Ontiveros y a Eduardo Gutiérrez la orientación y comentarios iniciales.
}

ISSN: 0212-8594 ISSN-e: 2340-2776.№ DOI: http://dx.doi.org/10.12795/rea.2015.i32.03 


\section{INTRODUCCIÓN.}

La preocupación por los avances recientes que la crisis se ha llevado por delante y por la pervivencia de debilidades estructurales constituye la conocida reflexión de arranque de Europa 2020 (Comisión Europea, 2010a, 5). Los objetivos generales de Lisboa 2000 siguen por tanto vigentes; esto es, convertir a la UE en la zona económica más dinámica y competitiva del mundo capaz de lograr un crecimiento económico sostenible con más y mejores empleos, más cohesión social y mayor sostenibilidad ambiental, sobre la base de una economía del conocimiento (Ministerio de Trabajo e Inmigración, 2010).

En ese sentido, la crisis económica ha reforzado el convencimiento sobre el papel del conocimiento y la innovación en la recuperación de la competitividad, en el crecimiento a largo plazo y a la hora de afrontar los importantes retos sociales existentes (cambio climático, envejecimiento, pobreza...) (OCDE, 2011a). Sin embargo, aunque las políticas de austeridad, como fórmula neoliberal de respuesta fundamental de Europa, puedan suponer un cierto alivio fiscal, las evidencias apuntan a que estas podrían estar erosionado al mismo tiempo las bases del crecimiento a largo plazo de países y regiones. Medidas como la desregulación laboral, los recortes o la privatización de servicios públicos, estarían exacerbando todo tipo de desigualdades sociales y geográficas, amenazando con destruir el principio de cohesión (Kitson, M. et al., 2011).

Así las cosas, desde comienzos de la crisis comienza a extenderse en nuestro país la idea acerca de la oportunidad del momento para corregir los desequilibrios de la economía (Pérez, F., 2011; Méndez, R., 2014) y plantear un cambio de modelo productivo, que inspire nuevos estilos de desarrollo local y regional (Jordá, R., 2010; Caravaca, I., 2014). Las estrategias de especialización inteligente (RIS3, en adelante) pueden marcar una hoja de ruta para este proceso de cambio. Hablamos de una aproximación bidireccional ("top-down" y "bottom-up") y dinámica, que persigue involucrar a los actores del territorio, sobre todo en la escala regional, en la definición de su propia visión y prioridades para el futuro (Foray, D. et al., 2011; Comisión Europea, 2012).

El objetivo primero del trabajo es estudiar el impacto de la crisis sobre el modelo productivo de las regiones españolas, con la mirada puesta en esa visión de la economía del conocimiento. En este sentido, los avances recientes en el seno del Grupo de Geografía Económica de la Asociación de Geógrafos Españoles (Albertos, J.M. y Sánchez, J.L., 2014) representan un excelente punto de partida para nuestro análisis. El objetivo segundo es mirar hacia el escenario de salida de la crisis (según desde qué perspectivas aún incierto en este año 2014), reflexionado al respecto a partir de las estrategias de RIS3 de las regiones españolas, teniendo en cuenta que se trata de un proceso que en muchos casos se inicia de forma reciente y que permanece abierto.

ISSN: 0212-8594 ISSN-e: 2340-2776.N№ DOI: http://dx.doi.org/10.12795/rea.2015.i32.03 
El trabajo se organiza de la siguiente forma. En la sección segunda se revisan algunos fundamentos teóricos importantes para la investigación, en especial sobre la equidad y el crecimiento (inteligente). En la sección tercera se analiza la transformación del modelo productivo durante la crisis, desde la perspectiva de las estructuras sectoriales y los llamados "clusters innovadores". En la sección cuarta se revisa el comportamiento de los sistemas regionales de innovación (SRI, en adelante) y se comenta el alineamiento de una muestra de estrategias de RIS3 con las capacidades empresariales y tecnológicas existentes. El trabajo termina recapitulando las principales conclusiones y planteando algunas líneas de trabajo a futuro.

\section{CONTEXTO TEÓRICO.}

\subsection{CRISIS, CRECIMIENTO Y EQUIDAD.}

Es un hecho repetido que la crisis económica ha ampliado los desequilibrios en el reparto de la riqueza y amenaza los logros alcanzados en Europa (Comisión Europea, 2010a). Aunque el crecimiento económico en la pasada década mejoró el nivel de vida global y permitió financiar la política social de muchos Estados, las desigualdades no han dejado de crecer, siendo todavía la pobreza y la exclusión social un grave problema para bastantes países de la UE (Comisión Europea, 2009). Como en el resto del mundo, Europa asiste al resurgimiento de los debates sobre crecimiento (eficiencia) y equidad, de larga tradición en la teoría económica (Galor, O., 2000; Stiglitz, J.E., 2012; Piketty, T., 2014) y con implicaciones fundamentales para la política regional (Cuadrado Roura, J.R., 2010).

Las últimas evidencias apuntan a que detrás del aumento de las desigualdades de renta durante la crisis se situarían las remuneraciones del trabajo, lo que a su vez se relaciona con diferencias en el capital humano y la capacidad productiva de los trabajadores (OCDE, 2011b). Siguiendo el análisis del profesor E. Ontiveros (2012), la globalización y la intensificación del cambio tecnológico, los procesos de offshoring y outsourcing, así como la extensión del comercio mundial de bienes y servicios intensivos en tecnología habrían forzado un cambio en la oferta de empleo (en términos de cualificaciones y perfiles profesionales). De este modo, el grado de asimilación y destrezas en nuevas tecnologías afectarían al nivel de remuneración alcanzado por lo trabajadores.

De forma paralela se apunta al factor de desigualdad que supone el aumento de las retribuciones en el sector financiero y su desigual distribución interna en los últimos años. Junto con ello, OIT (2012) apunta al impacto de los ajustes en el empleo, que se han cebado con los trabajadores de menor cualificación, así como al descenso de la calidad del empleo. Incluso así, hay quien sostiene la necesidad de cierto grado de desigual para un correcto funcionamiento económico; argumento utilizado para cuestionar el papel de las políticas redistributivas en relación con supuestas "filtraciones de eficiencia" (Champernowne, D.G. and Cowell, F.A., 1998). En este 
sentido, los análisis de A. Rodriguez-Pose and V. Tselios (2010) confirman lo complejo y en ocasiones contradictorio de los efectos de la desigualdad sobre el desarrollo regional.

Con todo, voces muy cualificadas subrayan en los últimos años los riesgos de la creciente desigualdad, no ya desde la perspectiva de la cohesión social ${ }^{3}$, sino del propio crecimiento económico; como se ha señalado, parece existir suficiente evidencia de que la "desigualdad no es rentable" (Ontiveros, E. 2011 y 2012; Stiglitz, J.E., 2012; Piketty, T., 2014). En esta línea se dirige el influyente trabajo del FMI preparado por A. Berg and J. D. Ostry (2011), donde se sostienen que la igualdad en la distribución de la renta constituye un elemento clave del crecimiento económico y de su sostenibilidad a largo plazo, por delante de la calidad del crecimiento, de las instituciones o la apertura exterior. De la misma forma, la desigualdad se relaciona con el riesgo de nuevas crisis financieras (Rajan, R., 2010) y de inestabilidad política, lo que afectaría directamente a la inversión internacional (Spence, M., 2012). Reorganizando las evidencias existentes, E. Ontiveros (2012) añade algunas otras consideraciones importantes en relación con los problemas que la desigualdad causa en la generación de capital social y confianza entre los actores económicos y con las instituciones; o con el papel de las políticas económicas en la consecución de una distribución más sostenible (fundamentalmente vía la educación, además de medidas en el mercado de trabajo o sistemas impositivos e impuestos).

En este estado de cosas, el cambio de patrón de crecimiento que encarna Europa 2020 busca garantizar la competitividad y el crecimiento futuro, al tiempo que un mejor reparto de la riqueza que refuerce la cohesión económica, social y territorial. Aunque la contribución de cara a sufragar los servicios del bienestar se hace menos explícita que en Lisboa 2000, se mantiene la aspiración de que futuras generaciones puedan disfrutar de ese modelo social "único" de Europa (Comisión Europea, 2010a). La fórmula no es nueva: situar la transformación productiva como motor de la competitividad y al mismo tiempo de la equidad ${ }^{4}$. En este caso, la transformación productiva se dirigiría a la consolidación de una economía basada en el conocimiento y la innovación, mediante una estrategia de crecimiento inteligente:

El crecimiento inteligente significa la consolidación del conocimiento y la innovación como impulsores de nuestro crecimiento futuro. Esto requiere mejorar la calidad de nuestra educación, consolidar los resultados de la investigación,

\footnotetext{
${ }^{3}$ La crisis ha significado un incremento de las desigualdades a nivel mundial (OCDE, 2011b). En el contexto de la UE se confirma el aumento de la población en riesgo de pobreza o exclusión social (Comisión Europea, 2013); en España el porcentaje de población por debajo del umbral de pobreza relativa pasó del 19,7\% en 2007 al 21,1\% en 2012 y del umbral de pobreza extrema del $4 \%$ en 2008 al 6,4\% en 2012 (Fundación FOESSA and Cáritas, 2012; Pitarch, M.D., 2014).

${ }^{4}$ Destacan en este sentido las propuestas de la CEPAL que perseguían la transformación productiva para asegurar una mayor prosperidad y equidad de los países latinoamericanos superada la década de los ochenta del pasado siglo (CEPAL, 1991).
}

ISSN: 0212-8594 ISSN-e: 2340-2776.№ DOI: http://dx.doi.org/10.12795/rea.2015.i32.03

REA 32 (2015): 49-75

http://editorial.us.es/es/revista-de-estudios-andaluces 
promover la innovación y la transferencia de conocimientos en toda la Unión, explotar al máximo las TIC y asegurarse de que las ideas innovadoras puedan convertirse en nuevos productos y servicios que generen crecimiento y empleos de calidad y que ayuden a afrontar los retos derivados de los cambios sociales en Europa y en el mundo (Comisión Europea, 2010a, 15).

Ahora bien, los expertos advierten que en el nuevo ciclo de la globalización, las oportunidades de la economía del conocimiento para UE disminuyen a medida que otras economías emergentes con menores costes sociolaborales se especializan en I+D y exportan productos de alto valor añadido, al tiempo que las TIC favorecen la deslocalización de tareas de alta cualificación y empleos relacionados con la Sociedad de la información (Ministerio de Trabajo e Inmigración, 2010). En cualquier caso, no se trata solo de asegurar la competitividad global; el crecimiento inteligente tiene retornos sobre la equidad y la cohesión. En primer lugar, talento, creatividad, conocimiento, habilidades y experiencia constituirían pilares fundamentales del nuevo modelo productivo (Rodríguez-Pose, A. and Tselios, V., 2010). La educación, en combinación con el avance de la Sociedad de la información, puede suponer una fuente de empleo en nuevos servicios y productos, además de actuar sobre las desigualdades salariales. Por otro lado, se pretende orientar el cambio estructural para fomentar oportunidades económicas y la innovación social como respuesta a los grandes retos para la sociedad en Europa y en el mundo (envejecimiento, desigualdad, impulso de una economía eficiente en el uso de recursos y baja en carbono...)

\subsection{LAS REGIONES ANTE EL RETO DE LA ESPECIALIZACIÓN INTELIGENTE.}

La oportunidad de definir un nuevo modelo de desarrollo en la salida de la crisis tiene un referente fundamental la especialización inteligente, elemento fundamental de la iniciativa Unión para la Innovación de Europa 2020 y de la reformada Política de Cohesión (Comisión Europea, 2010b). El concepto, que tiene un origen académico próximo a la "ventaja regional construida" y los enfoques evolucionistas en geografía económica (Foray, D. et al., 2011; Boschma, R., 2014), habría visto aumentado su interés en el contexto de la actual crisis, quedando recogido en distintas agendas políticas (OCDE, 2011a).

Desde el convencimiento que no puede haber una solución única, la especialización inteligente busca reducir la vulnerabilidad regional ante las condiciones económicas cambiantes, mediante una suerte de cambio estructural a partir de algunos procesos (no excluyentes) como: transición (de un sector existente a otro nuevo), modernización (mejora tecnológica de una industria existente, destacando las llamadas "tecnologías facilitadoras esenciales"), diversificación (descubrimiento de sinergias potenciales entre actividades existentes o con alguna nueva) y fundación radical de un nuevo dominio (mediante la aplicación de I+D+i a actividades de bajo crecimiento y un proceso de emprendimiento). Todo ello se reflejaría en una visión y una serie de prioridades regionales. 
De forma sintética, en el centro de la estrategia de RIS3 se sitúa el llamado proceso de "descubrimiento emprendedor" (Hausmann, R. and Roderik, D., 2003). En el mismo, corresponde a los emprendedores descubrir y decidir qué dominios de especialización son prioritarios, identificando oportunidades tecnológicas y nichos de mercado, a partir del análisis de estructuras productivas y capacidades del conocimiento distintivas de cada región (principalmente clusters, redes y empresas líderes), además de efectos espaciales y spillovers, dinámicas de emprendimiento y posicionamiento internacional. En este contexto, el emprendedor se define en un sentido amplio como individuos y organizaciones (por ejemplo, inversores, empresas, organizaciones de educación superior...) que poseen el conocimiento tecnológico, científico y del mercado necesario. Según la propia Comisión Europea (2012), la principal dificultad sería conseguir que dicha visión sea ambiciosa pero al mismo tiempo creíble; audaz y lo suficientemente amplia para acomodar de forma realista las prioridades y las trayectorias de desarrollo específicas, además de contemplar los retos relevantes para la sociedad. Se trata al mismo tiempo de encontrar soluciones en la diversificación que tengan en cuenta las condiciones socio-económicas y los contextos históricos locales ("regional embeddedness") y también las redes, especialmente de innovación, que articulan el espacio regional y lo conectan a nivel internacional.

El carácter bidireccional aludido al comienzo se plasma en la proposición de algunas tipologías regionales para orientar la elección de prioridades. La principal de ellas, aunque no la única, aproxima la dimensión del crecimiento inteligente a través de la propuesta de la OCDE (2011a) sobre: hubs de conocimiento, zonas de producción industrial y regiones no basadas en ciencia y tecnología. Esto da lugar a tres familias de estrategias posibles: (i) desarrollo basado en las ventajas actuales, con impulso a la ciencia o tecnología; (ii) apoyo a la transformación económica (por reconversión o identificación de una nueva frontera); (iii) reducción de la distancia ("catching-up") mediante la creación de capacidades basadas en conocimiento (Figura 1).

El hecho fundamental es que resulta imposible conocer a priori los resultados, siendo el propio proceso político, diseñado para asegurar la participación de los actores y que estos hagan suya la visión de la región a largo plazo, tanto o más importante que los resultados obtenidos (Boschma, R., 2014). Se apuesta así por un modelo de "cuádruple hélice", que incorpora a la industria, las instituciones de educación superior e investigación y el gobierno, además de un cuarto componente por el lado de la demanda (consumidores y usuarios de la innovación, organizaciones sin ánimo de lucro y otros actores de la sociedad civil, además de expertos externos y evaluadores); también por esquemas de gobernanza multinivel, flexibles y adaptados a la región (nivel clave para la concertación de las políticas europeas, nacionales y regionales favorecedoras del cambio estructural ${ }^{5}$ ); y por un "liderazgo colaborativo", haciéndose

\footnotetext{
5 RIS3 busca el alineamiento con las estrategias nacionales de investigación y educación (o regionales según grado de descentralización de competencias) y con la Política de Cohesión. La "condicionalidad ex-ante" obliga a que cada país/región defina su estrategia de desarrollo y las necesidades financieras
}

ISSN: 0212-8594 ISSN-e: 2340-2776.No DOI: http://dx.doi.org/10.12795/rea.2015.i32.03

REA 32 (2015): 49-75

http://editorial.us.es/es/revista-de-estudios-andaluces 
Figura 1. Estrategias de innovación según intensidad de conocimiento del tejido productivo regional.

\begin{tabular}{|c|c|c|c|}
\hline \multirow[b]{2}{*}{ Type of region } & \multicolumn{3}{|c|}{ Main strategy } \\
\hline & $\begin{array}{l}\text { Building on current } \\
\text { advantages (science } \\
\text { push/technology-led or } \\
\text { a mix) }\end{array}$ & $\begin{array}{l}\text { Supporting socio- } \\
\text { economic } \\
\text { transformation }\end{array}$ & $\begin{array}{c}\text { Catching-up: Towards } \\
\text { the creation of } \\
\text { knowledge-based } \\
\text { capabilities }\end{array}$ \\
\hline \multicolumn{4}{|c|}{ Knowledge hubs } \\
\hline \multicolumn{4}{|l|}{$\begin{array}{l}\text { Knowledge and } \\
\text { technology hubs }\end{array}$} \\
\hline \multirow{2}{*}{\multicolumn{4}{|c|}{$\begin{array}{l}\text { Knowledge-intensive } \\
\text { city/capital districts }\end{array}$}} \\
\hline & & & \\
\hline \multicolumn{4}{|l|}{$\begin{array}{l}\text { S\&T intensive production } \\
\text { regions }\end{array}$} \\
\hline \multicolumn{4}{|l|}{ Skill intensive regions } \\
\hline \multicolumn{4}{|l|}{$\begin{array}{l}\text { Medium-tech } \\
\text { manufacturing and service } \\
\text { providers }\end{array}$} \\
\hline \multicolumn{4}{|l|}{$\begin{array}{l}\text { Traditional manufacturing } \\
\text { regions }\end{array}$} \\
\hline \multicolumn{4}{|c|}{ Non-S\&T-driven regional systems } \\
\hline \multicolumn{4}{|l|}{$\begin{array}{l}\text { Service-led and natural } \\
\text { resource-based regions }\end{array}$} \\
\hline \multicolumn{4}{|l|}{$\begin{array}{l}\text { Structural inertia or de- } \\
\text { industrialising regions }\end{array}$} \\
\hline $\begin{array}{l}\text { Primary sector intensive } \\
\text { regions }\end{array}$ & & & \\
\hline
\end{tabular}

Legend: Omain priority; $\mathbf{O}$ strategic choice; $O$ low priority.

Fuente: Foray, D. et al. (2011).

hincapié en la necesidad de evitar que la estrategia pueda ser dominada por grupos de interés tradicionales (lobbies), elites o simplemente por actores regionales principales buscando aumentar sus rentas (McCann, P. and Ortega-Argiles, R., 2013). Para evitar estos problemas se plantea un proceso abierto e inclusivo, con intervención de moderadores externos, y fuertemente monitorización.

\section{EL CAMBIO SECTORIAL DURANTE LA CRISIS: ¿HACIA UNA ECONOMÍA DEL CONOCIMIENTO?}

El estallido de la crisis puso de manifiesto los desequilibrios del modelo productivo español, forjado desde mediados de la década de los noventa; en relación con aspectos como la baja productividad, el déficit exterior, el endeudamiento (sobre todo privado) $y$, fundamentalmente, el sobre-dimensionamiento de los sectores de construcción y turismo, frente a la debilidad de las actividades intensivas en conocimiento y en general del sistema de I+D+i (Naredo, J.M., 2009; Pérez, F., 2011; Rodríguez, E. and López, I., 2011; Méndez, R. 2014). Al mismo tiempo, expertos e instituciones de todo tipo han subrayado la ausencia durante aquellos años de 
crecimiento de auténticas políticas de transformación productiva; industriales, del sector energético, de investigación y formación (Fundación 10 de Mayo, 2011; Puig, A., 2011; Alonso, J.L., 2014). Y que hoy se vincula con algunos problemas que arrastra el país, empezando por el abandono escolar, la sobrecualificación o la mala calidad del empleo (Pérez, F., 2011), todos ellos de vital importancia desde la perspectiva apuntada del crecimiento con equidad. A todo lo anterior habría que sumar déficits político-institucionales que afectarían a la regulación económica y, por tanto, a la capacidad de hacer efectivo el cambio de modelo productivo (Costas, A. 2010; Romero, J. et al., 2014).

Desde esa visión (¿retórica?) de la crisis como oportunidad, cabe preguntarse por el rumbo seguido por las regiones españolas, primero desde la perspectiva de las estructuras sectoriales que han caracterizado al conjunto. En este sentido, partimos de la consideración que su resistencia al cambio habría sido en principio grande (Sánchez, J.L., 2014), si bien en el detalle por ramas de actividad sí se advierten cambios significativos (Alonso, J.L., 2014).

La utilización de los registros de afiliación a la Seguridad Social (a fecha 31 de diciembre de 2006 y 2012) nos permite aproximar el posible cambio en el "mix" sectorial de las regiones españolas durante la crisis, al tiempo que detectar especializaciones y masas críticas (tal como proponen las estrategias de RIS3). Aunque la fuente no incluye la información sobre trabajadores desempleados ni funcionarios, el principal escollo metodológico resulta del cambio en el sistema de clasificación de actividades $^{6}$. En el Anexo I se recoge la adaptación de los datos de 2012 (CNAE 2009) y 2006 (CNAE93 rev. 1). Siendo conscientes de los problemas que ello puede causar a la hora de comparar los datos, en el estudio se analizan preferentemente los cambios relativos al nivel de especialización y solo en cuanto al peso regional con datos agregados ${ }^{7}$.

Nuestro estudio toma como punto de partida el análisis shift-share realizado por el profesor J. M. Albertos (2014), donde se concluye que el efecto asociado a la estructura sectorial resultaría menos determinante que las ventajas competitivas locacionales (efecto regional) a la hora de explicar el crecimiento del VAB regional

\footnotetext{
${ }^{6}$ A lo largo de la serie se han producido otros cambios significativos. Un primer cambio surge de la bonificación del alta de los trabajadores de los servicios de la dependencia en el Régimen General de la Seguridad Social, impulsada por el último gobierno socialista (2008) y que las medidas de ajuste (2010) dejaron sin efecto. El otro afectaría a los empleados del servicio doméstico (2012). Ambos suponen una modificación al alza del empleo en los subsectores de servicios sociales y de actividades de los hogares respectivamente.

${ }^{7}$ La publicación en el caso de alguna Comunidad Autónoma de la información en ambos sistemas CNAE durante los primeros meses de 2009 ha permitido confirmar que los sesgos introducidos por la reclasificación tienden a descender y a compensarse al agregar la información. Tomando el caso de Aragón como ejemplo, la diferencia para el conjunto de la economía del conocimiento podría estar en torno a un $-6 \%$ en 2012 respecto de 2009.
}

ISSN: 0212-8594 ISSN-e: 2340-2776.N№ DOI: http://dx.doi.org/10.12795/rea.2015.i32.03 
entre 2008 y 2012. Sobre esta evidencia se propone una primera tipología regional que resulta de interés para organizar nuestros resultados de la Figura 2:

Un primer grupo de regiones, con un crecimiento por encima del resto, contaría con un efecto positivo tanto estructural como regional. Es el caso de las dos principales aglomeraciones urbanas del país, donde el dinamismo y la competitividad de su tejido empresarial, beneficiado por importantes economías de aglomeración, resultan tanto - más importantes que su perfil sectorial (diversificado, terciarizado y sin especialización en construcción, ni antes ni durante la crisis). De este modo, Madrid arranca con especializaciones industriales ya tradicionales (edición, papel y artes gráficas, material eléctrico y electrónico...), pero sobre todo en el terciario avanzando y los servicios públicos. Cataluña presenta especialización en todo tipo de industrias (alimentación, textil, material eléctrico y electrónico, material de transporte, entre otras) y en varias ramas de servicios, destacando el terciario avanzado y los servicios públicos. En ambos casos, los principales cambios apuntan a la pérdida de peso de alguna rama industrial y el aumento de servicios empresariales y públicos. Formarían parte del grupo otras dos regiones: Castilla León, que parte de especialización en agricultura y ganadería y varias industrias tradicionales (alimentación, extractivas, madera...), además de material de transporte, energía, construcción y el conjunto de servicios públicos; y Baleares, donde aparte de manufacturas tradicionales, como cuero y calzado, destacan las ramas de energía, construcción, comercio, hostelería y los servicios públicos en general. Con la crisis se advierte el ajuste de la construcción, además del dinamismo del cluster turístico balear (en relación con el tamaño de las empresas y sus estrategias de internacionalización) y de la industria castellanoleonesa (por factores de competitividad similares al caso anterior o más directamente vinculados con el SRI).

Según el mencionado trabajo, un segundo grupo presentaría un efecto estructural negativo muy débil, pero que es compensado por otro regional positivo. Esto se relacionaría con un crecimiento más limitado de la burbuja inmobiliaria y un ajuste posterior en construcción menos intenso, junto con el mejor comportamiento relativo de algunos otros sectores clave; como industria manufacturera o turismo (como en las dos últimas regiones mencionadas), además de actividades agrícolas o servicios empresariales. Destaca así la fortaleza del sector industrial de Navarra y País Vasco (edición, maquinaria y equipo mecánico, material eléctrico y electrónico, entre otras actividades), junto con energía, educación y sanidad en el primer caso; e intermediación financieras y servicios públicos en el segundo. Los cambios aquí son limitados, evidenciándose la mayor resistencia de la industria y la buena marcha de los servicios a las empresas (País Vasco). La Rioja y Aragón comparte estos rasgos de nula especialización en construcción pero sí en distintas ramas industriales, las cuales por otro lado parecen sufrir de forma más acusada el impacto de la crisis (sobre todo en Aragón), al tiempo que se refuerza el posicionamiento en torno a hostelería, educación y sanidad. En cambio, Galicia y Cantabria sí partían con especialización en construcción, además de actividades intensivas en recursos naturales y distintas ramas 
Figura 2. Especialización de las regiones españolas en 2006 y 2012.
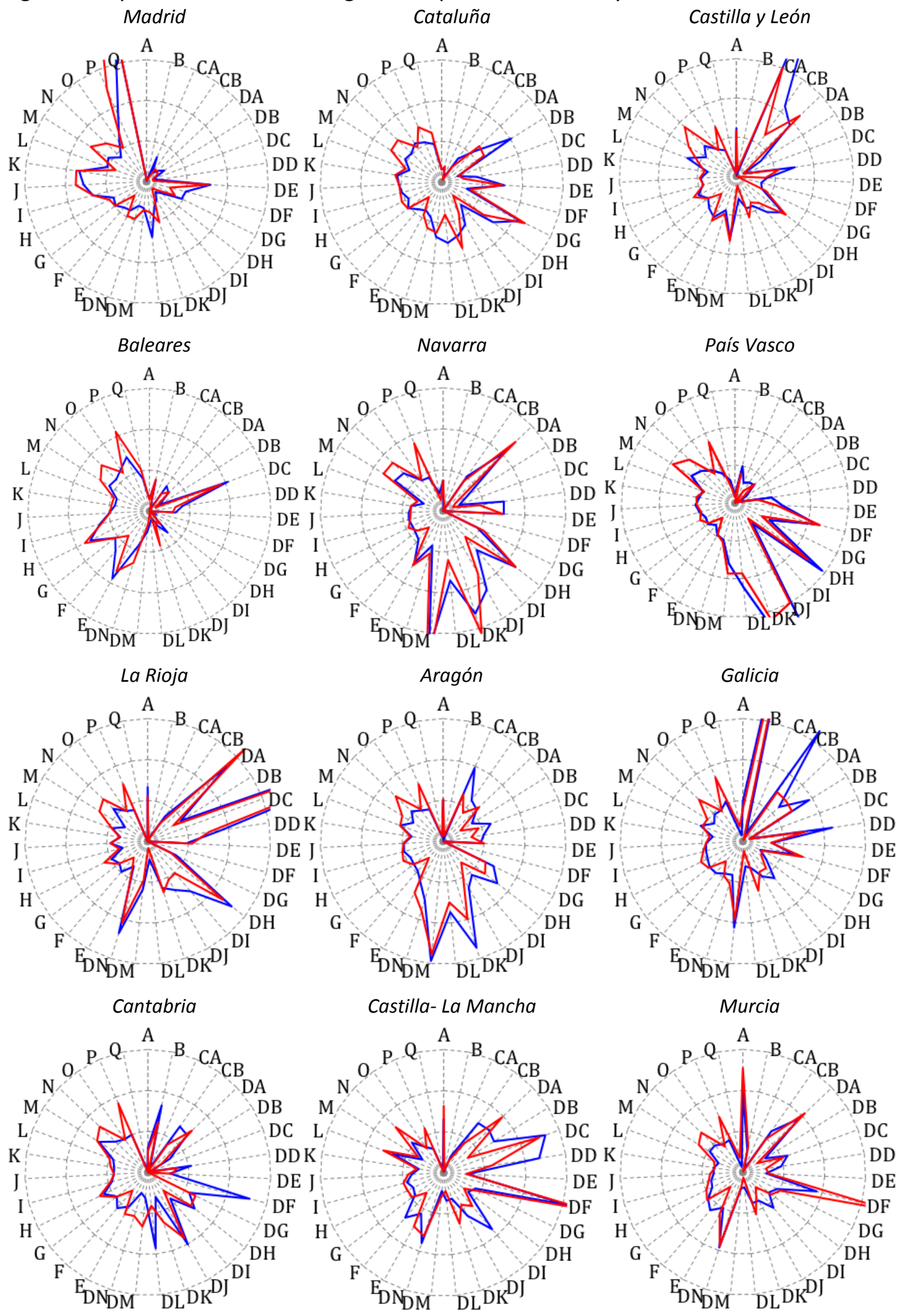

ISSN: 0212-8594 ISSN-e: 2340-2776.N№ DOI: http://dx.doi.org/10.12795/rea.2015.i32.03

REA 32 (2015): 49-75

http://editorial.us.es/es/revista-de-estudios-andaluces 
Figura 2. Especialización de las regiones españolas en 2006 y 2012 (continuación).
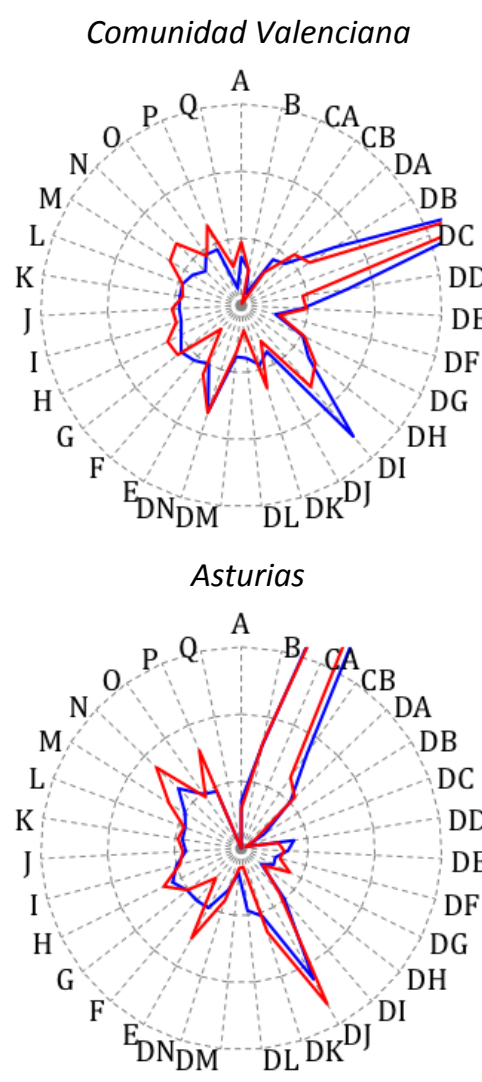

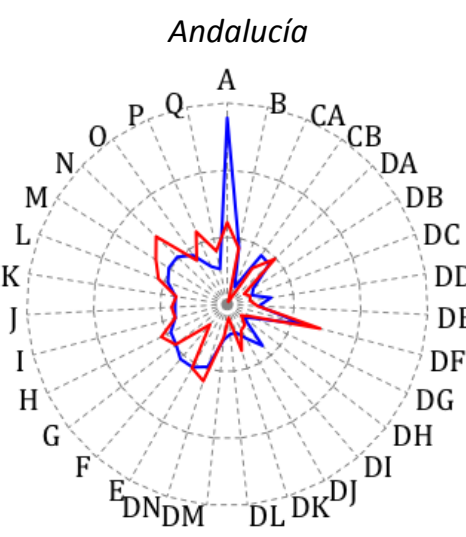

\section{Canarias}

Canarias
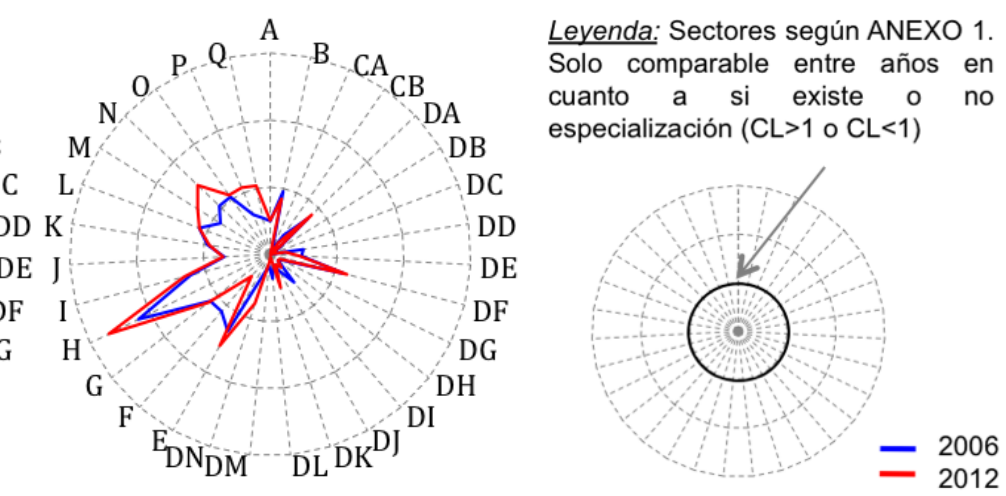

Fuente: Elaboración propia a partir de Tesorería General de la Seguridad Social.

industriales y de servicios. Aquí la crisis ha supuesto la caída de la construcción, una evolución dispar de la industria y en general un reforzamiento de los servicios, sobre todo de los públicos.

Un tercer grupo se vería afectado por unos efectos estructural y regional desfavorables, coincidiendo con algunos de los niveles de especialización en construcción más altos del país, lo que por otro lado ha sido relacionado con el grado de sensibilidad de las regiones a la crisis (Daher, A., 2013; Sánchez, J.L., 2014), y graves déficits de competitividad empresarial (Albertos, J.M., 2014). De este modo, CastillaLa Mancha arranca con especialización en agricultura y manufacturas tradicionales vinculadas a algunos sistemas productivos locales (alimentación, textil, cuero y calzado, madera), además de refino de petróleo, construcción, administración pública y sanidad. Murcia y la Comunidad Valenciana comparten muchos rasgos de esa estructura productiva, a la que añaden especialización en actividades relacionadas con el turismo y, en el segundo caso, una mayor diversificación de la industria. Con la crisis se repite en los tres casos la caída de la construcción y de varias industrias tradicionales, si bien se refuerzan otras de mayor intensidad tecnológica, algunos servicios basados en conocimiento (Valencia) y, sobre todo, los servicios públicos

ISSN: 0212-8594 ISSN-e: 2340-2776.N№ DOI: http://dx.doi.org/10.12795/rea.2015.i32.03 
(salvo educación en Castilla-La Mancha). Por su parte, Andalucía y Extremadura arrancan especializadas en unas pocas actividades, como agricultura, ganadería y otras intensivas en recursos naturales en el segundo caso (energía, alimentación, madera y corcho...). Junto con ello, destaca la especialización inicial en construcción y servicios públicos. En un contexto de marcada estabilidad, los principales cambios apuntarían a la pérdida de especialización en construcción y el refuerzo de la Administración y los distintos servicios públicos. Asturias, que se sumaría a este grupo rompiendo el patrón espacial del sur peninsular, aparecía más diversificada entre actividades intensivas en recursos naturales (extractivas, energía), manufacturas tradicionales en la región (metalurgia y maquinaria), construcción y diversos servicios públicos y privados. La caída de la construcción sería el principal cambio en la estructura productiva.

Finalmente, Canarias sería la única región que presenta un efecto regional negativo y un efecto estructural positivo, relacionado con su orientación turística (y que como en el caso de Baleares resulta determinante). Así, destaca la especialización inicial en hostelería, comercio y transporte, además de refino de petróleo, construcción, Administración y servicios públicos. La crisis ha supuesto la pérdida de especialización en construcción y el refuerzo de los servicios públicos en general.

En este punto, cabe preguntarse por el balance de todos estos cambios desde una perspectiva sectorial de la economía del conocimiento. En este sentido, de acuerdo con lo planteado en trabajos anteriores cabría diferenciar en principio seis clusters innovadores (Méndez, R. and Sánchez-Moral, S., 2011, Sánchez-Moral, S. et al., 2014): (1) Industrias de alta intensidad tecnológica (producción de TICs y otras actividades como industria farmacéutica, aeronáutica); (2) Actividades financieras y de seguros; (3) Servicios a las empresas intensivos en conocimiento (actividades informáticas, I+D y todo tipo de servicios de consultoría, asesoramiento, publicidad, etc.); (4) Telecomunicaciones; (5) Educación, sanidad y servicios sociales; (6) Industrias culturales (producción de bienes culturales como edición o audiovisuales, además de artes escénicas, museos y bibliotecas) (ver Anexo I).

En el año 2012 estos clusters en su conjunto alcanzaron los 5.278.939 empleos, pero con un reparto interno muy desigual: con educación, sanidad y servicios sociales (13\%) así como servicios a las empresas intensivos en conocimiento $(12,1 \%)$ muy por delante del resto (Cuadro 1). Por otro lado, su distribución regional, donde destacan Madrid (22,63\% del empleo total), Cataluña (19,50\%), Andalucía (13,70\%), Comunidad Valenciana $(8,63 \%)$ y País Vasco $(5,99 \%)$, confirma la atracción ejercida por las grandes áreas urbanas ante el stock disponible de recursos tangibles e intangibles para su desarrollo (Musterd, S. and Murie, A., 2010; Méndez, R. and Sánchez-Moral, S., 2011). Su comportamiento durante la crisis habría sido mejor que el resto de actividades $(+3,83 \%$ frente a $-19,41 \%)$, reforzando su presencia en el conjunto de la economía (más de 5 puntos porcentuales). Ahora bien, dentro de esta evolución se esconden trayectorias regionales contrastadas según clusters innovadores:

ISSN: 0212-8594 ISSN-e: 2340-2776.N№ DOI: http://dx.doi.org/10.12795/rea.2015.i32.03 
- Las industrias de alta intensidad tecnológica sería el cluster innovador más afectado por la crisis, lo que puede asociarse a cierres, desinversiones y deslocalizaciones de las multinacionales del sector (Méndez, R., 2013). La caída del empleo afectaría prácticamente a todas las regiones, siendo esta especialmente intensa en Baleares y Canarias.

- Tres clusters presentan una evolución menos negativa. En cuanto a las industrias culturales, afectadas en estos años por medidas fiscales que han penalizado su consumo y por una reducción de las ayudas públicas, destacan de forma negativa los casos de Murcia y Comunidad Valenciana. Las actividades financieras solo presentan una tendencia positiva en Madrid, frente al ajuste generalizado fruto de la reestructuración del sistema financiero español, especialmente en regiones con entidades financieras locales afectadas (Canarias, Baleares, Cataluña, Navarra, entre otras). Por su parte, los servicios a las empresas intensivos en conocimiento se habrían visto lastrados por los problemas del sector financiero, así como por la caída de la demanda del sector industrial y de las administraciones públicas. Esto parece haber afectado a Madrid y Barcelona y, sobre todo, a Valencia; pero no así al País Vasco, lo que de nuevo cabe relacionar con la fortaleza de su tejido empresarial y la calidad del SRI. También la trayectoria de Castilla y León resultó más favorable que el resto.

- Las telecomunicaciones presentan una evolución positiva del empleo durante la crisis, destacando su comportamiento en La Rioja, Murcia o Valencia. Como hemos podido comprobar, el cluster de educación, sanidad y servicios sociales habría experimentado el mayor incremento. Sin perder de vista los efectos estadísticos comentados, varios hechos parecen estar detrás de este incremento: la propia inercia de la variable empleo, que reflejaría crecimientos asociados al desarrollo del Estado del Bienestar en los años anteriores a la crisis, así como algunas medidas que buscaron reactivar la economía y amortiguar su impacto en un primer momento. Por otro lado, hablamos de empleo público y privado y, por tanto, no puede descartarse el impacto de estrategias de privatización "por partes" del Estado del Bienestar (Romero, J. et al., 2014) $)^{8}$.

\section{EL COMPORTAMIENTO DE LOS SISTEMAS REGIONALES DE INNOVACIÓN Y EL ALINEAMIENTO DE LAS ESTRATEGIAS DE RIS3.}

Aunque en los últimos años resultó habitual el señalar al sobre-dimensionamiento de la construcción y las actividades de servicios de baja productividad como el principal problema del modelo productivo español, lo cierto es que en el conjunto de la

\footnotetext{
${ }^{8}$ Las competencias que se refieren a las actuaciones del sector público relativas al Estado del Bienestar están transferidas a las Comunidades Autónomas (a excepción de las pensiones y otros subsidios). Según sus presupuestos liquidados, la variación 2009-2012 del gasto conjunto en Sanidad, Educación y Cultura fue del -11,4\% y en Servicios Sociales y Promoción Social del -3,8\% (Romero, J. et al., 2014).
}

ISSN: 0212-8594 ISSN-e: 2340-2776.№ DOI: http://dx.doi.org/10.12795/rea.2015.i32.03

REA 32 (2015): 49-75

http://editorial.us.es/es/revista-de-estudios-andaluces 
economía se advierten importantes debilidades competitivas. Detrás de ello estarían algunos déficits internos, en relación sobre todo con el tamaño empresarial, el perfil del emprendedor y la cualificación de los directivos, así como con el uso de activos del conocimiento o el grado de internacionalización; además de condicionantes del entorno, como las dificultades de acceso a la financiación, las relaciones laborales (según los directivos) o los problemas burocráticos (Pérez, F., 2011).

Muchas de esas variables guardan relación con las características y el funcionamiento de los sistemas regionales de innovación. Varios autores coinciden en señalar la escasa repercusión de los mismos sobre el crecimiento, apuntando algunos aspectos que interactuarían con algunas debilidades estructurales de la economía española ya apuntadas (Navarro Arancegui, M. and Gibaja Martins, J.J., 2009; Ondategui, J.C., 2008; Jordá, R. et al., 2014): i) Reducidas dimensiones y falta de masa crítica de empresas innovadoras que generen sinergias entre sí y con otros actores; ii) dependencia del sector público, lo que aumenta su vulnerabilidad ante la actual crisis; iii) desigual desarrollo y funcionamiento de los agentes y actores, lo que viene determinado por la desigual calidad de los respetivos SRI.

En ese sentido, el trabajo de Jordá et al. (2014), basado en indicadores sobre inputs en I+D+i, entorno socioeconómico y outputs científicos y económicos, nos ofrece una caracterización de los SRI y una perspectiva inédita del impacto de la crisis en su funcionamiento. De forma sintética, cabe destacar los siguientes aspectos de su diagnóstico:

- Se confirma la existencia de cuatro "SRI de excelencia": País Vasco y Navarra centrados en el subsector empresarial $(11,27 \%$ de empresas innovadoras, $13,49 \%$ del gasto en innovación empresarial, $12,60 \%$ de la inversión en I+D); mientras que Madrid y Cataluña se orientarían hacia la alta tecnología (37,68\% de empresas innovadoras, 59,32\% del gasto en innovación empresarial, 48,40\% de la inversión en I+D). Estos SRI cuentan con las mayores capacidades empresariales (industrias de alta intensidad tecnológica y servicios avanzados intensivos en conocimiento) y científico-tecnológicas (destacando la concentración de recursos públicos en Madrid).

- Un segundo grupo lo formarían "SRI con susbistemas desarticulados" (46,29\% de empresas innovadoras, $26,40 \%$ del gasto en innovación empresarial, 36,60\% de la inversión en I+D), diferenciándose según el mayor peso de la financiación privada en I+D (Aragón, Galicia, Castilla y León, La Rioja), pública (Andalucía, Cantabria, Comunidad Valenciana, Extremadura) o mixta (Asturias, Castilla-La Mancha, Murcia). Pese a las mayores limitaciones y desequilibrios de los SRI, también aquí se identifican algunos nichos de excelencia; por ejemplo en Castilla y León, derivado de la competitividad exterior de algunas industrias de alta-media intensidad tecnológica y de su sector de servicios avanzados; en Andalucía, en relación con sectores de alta-media intensidad tecnológica y la red de parques y centros tecnológicos asociada a clusters estratégicos (aeronáutica, biotecnología, energía solar...); en la Comunidad Valenciana o La 
Cuadro 1. Peso y evolución de las industrias intensivas en conocimiento, 2006-2012.

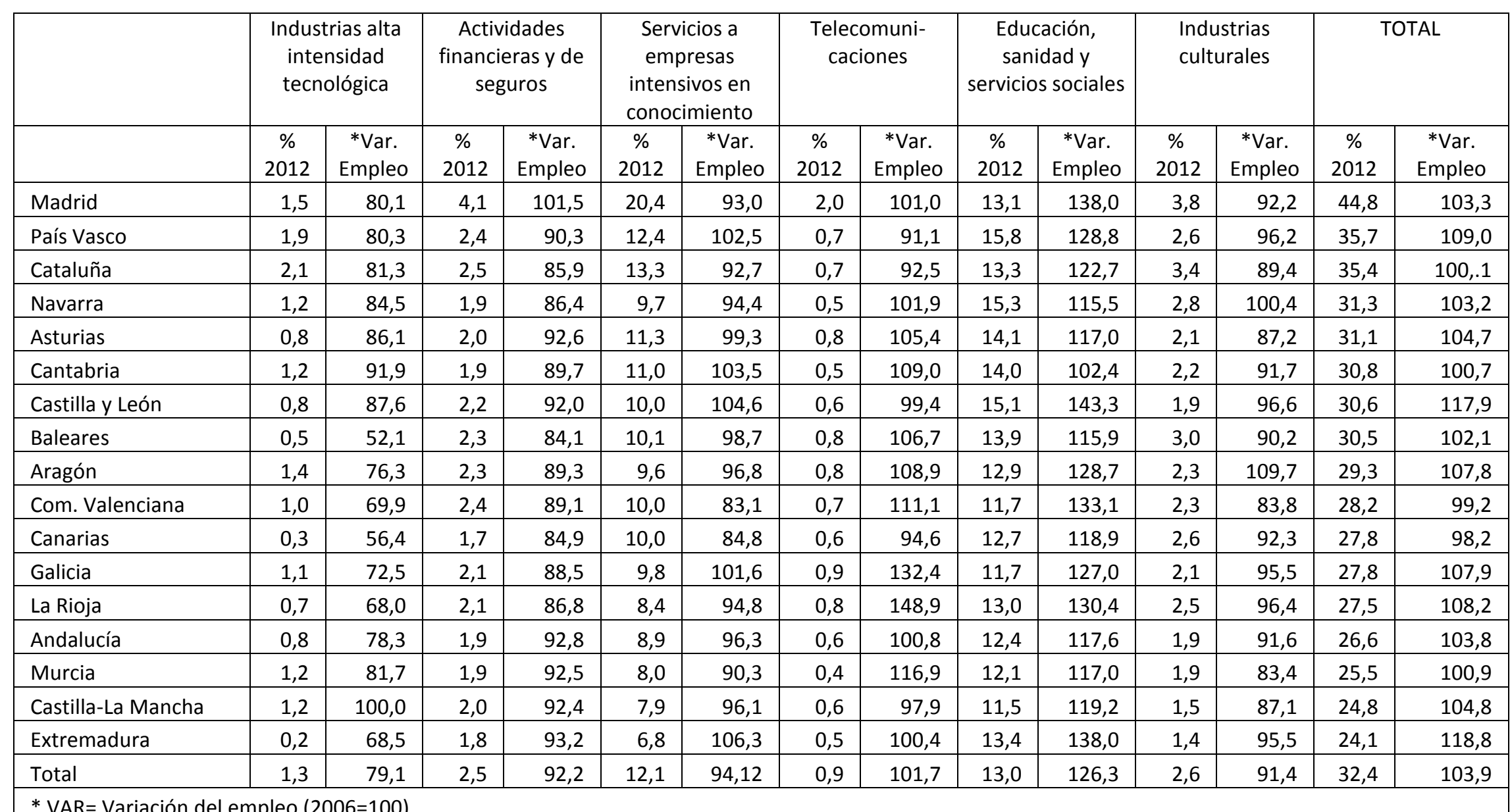

* VAR $=$ Variación del empleo $(2006=100)$

Fuente: Elaboración propia a partir de Tesorería General de la Seguridad Social.

ISSN: 0212-8594 ISSN-e: 2340-2776.N№ DOI: http://dx.doi.org/10.12795/rea.2015.i32.03

REA 32 (2015): 49-75

http://editorial.us.es/es/revista-de-estudios-andaluces 
Rioja, por su capacidad científico tecnológica (patentes a nivel internacional, publicaciones científicas, sistema educativo superior...).

- Finalmente se identifica a Baleares y Canarias como "elementales SRI insulares sostenidos por el sistema público" (4,65\% de empresas innovadoras, 0,80\% del gasto en innovación empresarial, $2,40 \%$ de la inversión en I+D). Pese a que sus indicadores de innovación se sitúan por debajo de la media nacional, aparecerían algunos nichos de excelencia en relación con la participación de centros canarios en redes internacionales de investigación.

Según Jordá et al. (2014), el impacto de la crisis se dejaría sentir en los subsistemas empresarial e institucional de todos estos SRI. De este modo, la mayor fortaleza del sistema empresarial en País Vasco y Navarra explicaría su distanciamiento del resto, con aumentos por ejemplo del gasto en I+D e innovación (pese a la caída de algunos indicadores de internacionalización o exportaciones). Esto no ocurre en Madrid y Cataluña, afectados por la caída de la inversión interna en I+D empresarial y del gasto en innovación ( $y$ del empleo en alta tecnología en el primer caso). Otra trayectoria negativa que se identifica corresponde a Aragón, en relación con la caída del gasto interno empresarial en I+D y del empleo en manufacturas de alta y media tecnología, asociado a desinversiones de grandes empresas, como en el resto del país. De hecho, en su grupo se suceden efectos negativos de este tipo. Junto con ello, la crisis económica y las políticas de ajuste habrían afectado a todos los subsistemas científicotecnológicos; con virulencia en Madrid y Cataluña, pero revistiendo especial gravedad allí donde la dependencia de los recursos públicos resulta mayor, como en los casos extremos de Baleares y Canarias, pero también de Andalucía o Extremadura, entre otros.

Ante todo este conjunto de evidencias cabe finalizar el trabajo preguntándonos hasta qué punto las estrategias de RIS3 se alinean con las capacidades de conocimiento y aprovechan la diversificación en los nichos de especialización existentes. Dado que nuestro objetivo es hacer una primera aproximación a una documentación cuya complejidad exigiría de análisis regionales pormenorizados, se ha decidido limitar dicho comentario a una muestra de tres regiones que, habiéndose sometido al proceso de evaluación externa de la Comisión Europea a través de la Smart Specialization Platform $\left(S^{3}\right)^{9}$, resultan representativas de los principales tipos de SRI comentados anteriormente (Cuadro 2).

Resulta visible la continuidad y coherencia de RIS3-Euskadi con las que han sido las directrices de la política económica vasca (desde el primer Plan de Competitividad en los noventa y sucesivos, el próximo Plan de Ciencia, Tecnología e Innovación, etc.), haciendo buena la reflexión de la Comisión al señalar que no se trata de que las regiones más avanzadas "empiecen desde cero". De hecho, de la documentación preliminar disponible ha trascendido la visión de la región que se ve a si misma como "precursora" de RIS3 en España. Las tres prioridades estratégicas de "Energía",

\footnotetext{
${ }^{9}$ http://s3platform.jrc.ec.europa.eu/home

ISSN: 0212-8594 ISSN-e: 2340-2776.№ DOI: http://dx.doi.org/10.12795/rea.2015.i32.03 
"Fabricación avanzada" y "Biociencias" parecen en principio bien alineadas con algunas de las especializaciones detectadas (energía, electrónica, bienes de equipo...) y con las capacidades tecnológicas y empresariales del SRI, al tiempo que se identifican oportunidades en sectores emergentes (salud humana), donde las tecnologías facilitadoras tendrían un papel especialmente importante. No podemos dejar de llamar la atención sobre esa cuarta prioridad de "Territorio", que incluye varios nichos en los que la región disfrutaría de un excelente posicionamiento internacional (por ejemplo en planificación y regeneración urbana). Por otro lado, la estrategia también refleja retos sociales como el cambio climático, el uso eficiente de los recursos o la urbanización.

RIS3-Aragón establece una visión un tanto general, a la que contribuirían tres prioridades planteadas con cierta dosis de "audacia", como sugiere la Comisión. Detrás de la prioridad "Conectividad" se sitúan sectores económicos claves para la región, donde se reconocen tanto capacidades tecnológicas como científicas y un buen posicionamiento internacional: logística y material de transporte. La "Eficiencia de los recursos" centraría su atención en los sectores de energía y agua, aprovechando el conocimiento acumulado en la región, al tiempo que "Bienestar y Calidad de Vida" agrupa los sectores agroindustrial, turismo y salud, buscando generar sinergias entre ellos y la generación de nuevos productos, procesos y tecnologías a través del emprendimiento. En este sentido, resulta visible la preocupación por resolver algunos problemas detectados en el SRI (apoyo a PYMEs, internacionalización, I+D y transferencia de conocimiento y tecnología), al tiempo que la sostenibilidad en el uso de los recursos o los procesos de envejecimiento de la población se perfilan como retos sociales a los que la estrategia apunta.

Finalmente, el documento preliminar de RIS-3 Canarias aporta una visión no exenta de cierta vaguedad, lo que parece traducirse en problemas a la hora de acotar las prioridades regionales. La primera de ellas establece el "Liderazgo inteligente del turismo", basado en la mejora de la competitividad del producto turístico y en una diversificación del cluster hacia actividades proveedoras y clientes. Menos preciso se presenta el intento de hacer de "Canarias, referente atlántico inteligente", capaz de dinamizar las relaciones humanas, culturales y comerciales entre Europa, África y América. Si en algunos casos parece existir relación con ámbitos de especialización regional (transporte, comunicaciones, energía y agua, educación y sanidad), en otros se puesta por abrir nuevas oportunidades vía la cooperación internacional, donde por otro lado se manifiesta de forma más clara la atención hacia los retos sociales. La "Valorización socioeconómica de la I+D" incide directamente en los déficits del SRI y busca aprovechar ámbitos de excelencia internacional (astrofísica y espacio, ciencias marítimo-marinas...). Finalmente, dentro de "Agenda digital" y "Crecimiento verde y sostenibilidad" se apuesta por tecnologías facilitadoras como TICs o biotecnologías. 
Cuadro 2. Ejemplos de estrategias de especialización inteligente (RIS3) en tres regiones españolas.

\begin{tabular}{|c|c|c|c|}
\hline Ámbito & Fechas clave & Visión & $\begin{array}{l}\text { Prioridades y Tecnologías Facilitadoras Esenciales } \\
\text { (KETs) }\end{array}$ \\
\hline $\begin{array}{l}\text { RIS3-Euskadi } \\
\text { _ "Innovation } \\
\text { Followers"* } \\
\text { _ "SRI de excelencia"** }\end{array}$ & $\begin{array}{l}\text { - Inicio: otoño } 2012 \\
\text { - Documento } \\
\text { preliminar: abril } \\
\quad 2013 \\
\text { - Presentación: enero } \\
\\
\quad 2015 \text { (prevista) }\end{array}$ & $\begin{array}{l}\text { Sin comunicar por el momento (región "precursora" de } \\
\text { RIS3 en España) }\end{array}$ & $\begin{array}{l}\text { 1. Energía } \\
\text { 2. Fabricación avanzada } \\
\text { 3. Biociencias } \\
\text { 4. Territorio } \\
\text { KETs: Materiales, Biotecnología, Nanotecnología, } \\
\text { Microtecnología, Fotónica, Tecnologías de } \\
\text { fabricación avanzada }\end{array}$ \\
\hline $\begin{array}{l}\text { RIS3-Aragón } \\
\text { - "Moderate } \\
\text { Innovators"* } \\
\text { - "SRI con subsistemas } \\
\text { desarticulados"** }\end{array}$ & $\begin{array}{l}\text { - Incorporación a } \mathrm{S}^{3} \text { : } \\
\text { febrero } 2012 \\
\text { - Presentación: } \\
\text { diciembre } 2013\end{array}$ & $\begin{array}{l}\text { "Aragón será en } 2020 \text { un territorio conectado y } \\
\text { sostenible reconocido a nivel internacional, mediante } \\
\text { la mejora de la conexión y movilidad, con el desarrollo } \\
\text { de prioridades en materia de sostenibilidad y mejora } \\
\text { de la calidad de vida de los habitantes, basado en una } \\
\text { diferenciación a través de las prioridades de } \\
\text { especialización inteligente y teniendo en cuenta los } \\
\text { desafíos, los retos, sus ventajas competitivas y su } \\
\text { potencial de excelencia" }\end{array}$ & $\begin{array}{l}\text { 1. Bienes y calidad de vida } \\
\text { 2. Conectividad } \\
\text { 3. Eficiencia de los recursos } \\
\text { KETs: Nanotecnología, Nuevos materiales, Nuevas } \\
\text { tecnologías de producción, TICs }\end{array}$ \\
\hline $\begin{array}{l}\text { RIS3-Canarias } \\
\text { - “Modest innovators"* } \\
\text { - “Elementales SRI } \\
\text { insulares sostenidos } \\
\text { por sector público"** }\end{array}$ & $\begin{array}{l}\text { - Incorporación a } \mathrm{S}^{3} \text { : } \\
\text { junio } 2012 \\
\text { - Documento } \\
\text { preliminar: mayo } \\
2013\end{array}$ & $\begin{array}{l}\text { "Canarias en } 2020 \text { será la referencia europea en el } \\
\text { Atlántico medio; como lugar de encuentro e } \\
\text { intercambio cultural y económico de tres continentes, } \\
\text { con una plena y efectiva integración en Europa y en los } \\
\text { flujos internacionales de ideas, personas, mercancías y } \\
\text { recursos financieros. Canarias constituirá una } \\
\text { magnífica plataforma para los negocios, la formación } \\
\text { y la promoción de nuevas tecnologías y conocimientos } \\
\text { vinculados a los retos del desarrollo humano, la salud y } \\
\text { la sostenibilidad medioambiental" }\end{array}$ & $\begin{array}{l}\text { 1. Liderazgo inteligente del turismo } \\
\text { 2. Canarias, referente atlántico inteligente } \\
\text { 3. Valorización socioeconómica de la I+D } \\
\text { 4. Agenda Digital (TIC) } \\
\text { 5. Crecimiento verde y sostenibilidad } \\
\text { KETs: TICs, Biotecnologías }\end{array}$ \\
\hline
\end{tabular}

*Según Regional Innovation Scoreboard (2014); **Según Jordá et al., 2014.

Fuente: Elaboración propia.

ISSN: 0212-8594 ISSN-e: 2340-2776.№ DOI: http://dx.doi.org/10.12795/rea.2015.i32.03

REA 32 (2015): 49-75

http://editorial.us.es/es/revista-de-estudios-andaluces 


\section{CONCLUSIONES.}

Desde el estallido de la crisis se viene reiterando la necesidad de un cambio en ese modelo de crecimiento del país, basado en el binomio turismo-construcción y sectores industriales de baja o media intensidad tecnológica, que aplazó durante años la aplicación de políticas de transformación productiva y que dificultó un desarrollo suficiente y equilibrado del sistema nacional de investigación. A partir de estos déficits y desequilibrios ( $y$ también de algunas fortalezas importantes como: dinamismo de multinacionales españolas, exportaciones, capital humano...) el país se enfrenta al reto de consolidar una economía basada en el conocimiento y la innovación, que asegure la competitividad y mayores niveles de cohesión social y territorial.

El planteamiento de las estrategias de especialización inteligente ha motivado nuestro intento de integrar en la escala regional las evidencias encontradas sobre el posible cambio de modelo productivo, desde una doble perspectiva. En primer lugar, en la aproximación al cambio en las estructuras sectoriales destaca el intenso ajuste laboral en el sector de la construcción, la caída menos pronunciada y desigual de las actividades industriales, según su naturaleza y condiciones territoriales específicas, y el aumento muy significativo del sector terciario, incluidos los servicios públicos (sobre todo en los primeros años de la crisis). Los procesos de cambio estructural sin duda son lentos, pero al descender a nivel de ramas de actividades algunas tendencias comienzan a dibujarse.

Ahora bien, como se ha repetido, el desenvolvimiento económico de las regiones depende de la eficiencia y capacidad de competir de las empresas. Esto guardaría a su vez relación con características internas (tamaño, internacionalización, capital humano) y condiciones del entorno, donde destaca la aparición de economías de aglomeración y efectos espaciales, así como el dinamismo de los propios SRI, dentro de los que una parte de esas empresas se incardinan junto con las instituciones. De los trabajos de J. M. Albertos (2014) aprendimos que este segundo grupo de factores resultaría en general más decisivo para las regiones; para las que cuentan con un "mix" sectorial de partida mejor alineado con la economía del conocimiento y desde luego para aquellas otras orientadas hacia actividades más tradicionales de bajo contenido tecnológico y/o en conocimiento.

Entre las primeras destacan Madrid y Cataluña, que escaparon de la especialización en actividades de construcción, generalizada en los años de crecimiento. Aunque la crisis habría afectado al empleo en algunas de sus ramas industriales de alta intensidad tecnológica y servicios avanzados a las empresas, todo apunta a que estas regiones avanzan hacia una consolidación de la economía del conocimiento, aprovechándose de la masa critica de empresas y actividades innovadoras y de unas economías de aglomeración de la máxima intensidad en el conjunto del país. Ello no impide que se detecten problemas en relación, por ejemplo, con procesos de deslocalización y desinversiones, caída del gasto en I+D empresarial o ajustes en los recursos públicos

ISSN: 0212-8594 ISSN-e: 2340-2776.N№ DOI: http://dx.doi.org/10.12795/rea.2015.i32.03

REA 32 (2015): 49-75

http://editorial.us.es/es/revista-de-estudios-andaluces 
destinados a los sistemas de ciencia-tecnología y educación. También en mejor situación aparecen País Vasco y Navarra, donde se asiste a un reforzamiento de varias ramas industriales y de los servicios avanzados a las empresas. Los factores de este mejor comportamiento relativo son diversos, destacando los relacionados con el SRI; tanto por el dinamismo del subsistema empresarial, como por la calidad y grado de implicación alcanzado por las instituciones. Dentro de este grupo de regiones avanzadas hemos podido aproximarnos a la estrategia Euskadi-RIS3, de la que se deducen diferentes planteamientos con vistas al proceso de cambio estructural y que apuntan hacia la modernización, transición e incluso fundación radical de nuevos dominios. Se aprovecharían así capacidades empresariales y tecnológico-científicas existentes, así como una dilatada historia de cooperación institucional, en un territorio pionero en el contexto nacional en la búsqueda de ventajas competitivas a través de la innovación.

Otras regiones españolas parten de una situación mucho más ambivalente; observándose cambios de diferente signo, un reparto muy desigual de las capacidades empresariales y/o tecnológicas según segmentos productivos, así como la aparición de déficits y desequilibrios en los respectivos SRI. Se evidencia en ellas el ajuste de la construcción (y en ramas afines) y el reforzamiento de los servicios públicos, al tiempo que los vaivenes de la industria confirman la influencia de esas claves competitivas específicas; visibles por ejemplo en el mejor comportamiento de algunos sistemas productivos locales tradicionales de La Rioja o Galicia ${ }^{10}$, pasando por los problemas del sector industrial en Aragón o el dinamismo de ramas de intensidad tecnológica mediaalta en Cantabria. En este sentido, la aproximación a la estrategia RIS3-Aragón parece confirmar que la aparición de nichos de especialización y áreas concretas de excelencia científico-tecnológica podría ser un caldo apropiado para el planteamiento de estrategias que persiguen una transformación socioeconómica vía la modernización tecnológica o la diversificación mediante sinergias. Incluso en esta región, que según qué diagnósticos se aproxima al grupo anterior, resulta visible la preocupación por mejorar el funcionamiento del SRI y la creación de capacidades de conocimiento.

En otras regiones, como Castilla-La Mancha, Murcia, Comunidad Valenciana, Andalucía - Extremadura, aunque la crisis también ha eliminado la especialización en construcción que caracterizó su modelo de desarrollo, no cabe hablar de grandes cambios en el mismo. El peso de la economía del conocimiento en estas regiones se sitúa a la cola del país (pese al aumento generalizado de los servicios públicos). Por otro lado, se observan problemas de competitividad y déficits en los respectivos SRI, que en algunos casos tienden además a ser muy dependientes de los recursos públicos. En el extremo de esta situación nos encontramos a Canarias. Más allá de cierta falta de precisión en la documentación preliminar sobre su estrategia de RIS3,

\footnotetext{
${ }^{10} \mathrm{El}$ comportamiento de los sistemas productivos locales en la actual crisis resulta un tema de particular interés, al que resulta necesario aproximarse a través de investigaciones monográficas (ver por ejemplo Salom, J. and Albertos, J. M., 2014).
}

ISSN: 0212-8594 ISSN-e: 2340-2776.№ DOI: http://dx.doi.org/10.12795/rea.2015.i32.03

REA 32 (2015): 49-75

http://editorial.us.es/es/revista-de-estudios-andaluces 
queda patente la apuesta por fortalecer el cluster turístico, favoreciendo estrategias de modernización y diversificación, mientras que se evidencia el esfuerzo por reducir la brecha existente respecto a otras regiones ("catching-up").

Concluimos planteando algunas reflexiones sobre nuestro trabajo, que no puede ocultar un planteamiento de partida panorámico y necesariamente sintético. De estas reflexiones surgen posibles líneas de avance en la investigación:

En primer lugar, la aproximación que se ha hecho aquí a la crisis y al posible cambio de modelo productivo resulta parcial, al centrarse sobre todo en las estructuras sectoriales. Sería necesario un análisis más profundo de otras variables económicas, algunas con un comportamiento complejo durante la crisis (productividad, exportaciones, endeudamiento), sin perder de vista otras dimensiones sociales fundamentales (sistema educativo, calidad del empleo, etc.). Al hilo de algunas de estas cuestiones, cabe preguntarse por la coherencia entre los discursos del crecimiento inteligente y la austeridad, lo que seguramente exige de un análisis más fino por fases dentro de la crisis.

Por otro lado, nos hemos aproximado a las especializaciones regionales, capacidades y "cuellos de botella" en los SRI, según proponen las estrategias de RIS3, pero es cierto que el estudio de los efectos espaciales de aglomeración y spillovers ha quedado en un segundo plano. Muchas evidencias apuntan a que las capacidades que se busca activar aparecen localizadas preferentemente en regiones urbanas capaces de generar economías de aglomeración, para contagiar posteriormente a territorios vecinos.

Finalmente, pensamos que la "especialización inteligente" puede ser una conceptualización relevante para los estudios de Geografía Económica. Por de pronto sus planteamientos han sido plenamente asumidos por la política regional Europea, insistiendo la Comisión en que no se trata tan solo de una estrategia de innovación centrada en el I+D. Desde la óptica del desarrollo territorial resultan de gran interés los mecanismos de gobernanza multinivel y de liderazgo colaborativo puestos en marcha para asegurar el desarrollo del proceso, del que cabe esperar un aumento del capital social y de la propia capacidad de resiliencia territorial. Todos ellos son aspectos en los que, junto con los fundamentos teóricos, habrá que seguir profundizando según se vayan conociendo los detalles de las estrategias de RIS3 aprobadas por las distintas regiones españolas.

\section{BIBLIOGRAFÍA}

Albertos J. M. (2014): "Estructuras sectoriales, territorio y diversidad de las trayectorias regionales con la crisis: un análisis shift-share espacial", in Albertos J. M. and Sánchez, J. L. (Coords.) Geografía de la crisis económica en España, pp. 231-262. Universidad de Valencia, Valencia. 
Albertos J. M. and Sánchez, J. L. (Coords.) (2014): Geografía de la crisis económica en España. Universidad de Valencia, Valencia, 770p.

Alonso, J.L. (2014): "Industria y crisis económica en España", in Albertos J. M. and Sánchez, J. L. (Coords.) Geografía de la crisis económica en España, pp. 141-172. Universidad de Valencia, Valencia.

Berg, A and Ostry, J.D. (2011): "Inequality and Unsustainable Growth: Two Sides of the Same Coin?", IMF Staff Discussion Note 11/08, IMF, Washington.

Boschma, R. (2014): “Constructing Regional Advantage and Smart Specialization: Comparison of Two European Policy Concepts", Papers in Evolutionary Economic Geography 13.22, CIRCLE-Lund University, Sweden.

Caravaca, I. (2014): "Desarrollo local frente a la crisis", Revista de Ciencias Sociales 25, pp. 95-115.

CEPAL (1991): El desarrollo sustentable: transformación productiva, equidad y medio ambiente. CEPAL ONU, Santiago de Chile, 154p.

Comisión Europea (2009): Growth, Jobs and Social Progress in the EU Report - A contribution to the evaluation of the social dimension of the Lisbon Strategy. The Social Protection Committee, September 2009. [En línea] <http://www.cedefop.europa.eu/en/news/3979.aspx.> [1 de junio de 2014].

Comisión Europea (2010a): Europa 2020: Una estrategia para un crecimiento inteligente, sostenible e integrador. COM, Bruselas, 38p.

Comisión Europea (2010b): Contribución de la Política Regional al crecimiento inteligente en el marco de Europa 2020. COM, Bruselas, 15p.

Comisión Europea (2012): Guide to Research and Innovation Strategies for Smart Specializations (RIS 3). DG Regio 2012, Luxemburgo, 130p.

Comisión Europea (2013): Octavo informe de situación sobre la cohesión económica, social y territorial. La dimensión regional y urbana de la crisis. COM, Bruselas [En línea] <http://eur-lex.europa.eu/LexUriServ/LexUriServ.do?uri=COM:2013:0463:FIN:ES:PDF> [1 de junio de 2014].

Costas, A. (Coord.) (2010) La crisis de 2008. De la economía a la política y más allá. Serie Mediterráneo Económico 18, Fundación Cajamar, Almería.

ISSN: 0212-8594 ISSN-e: 2340-2776.№ DOI: http://dx.doi.org/10.12795/rea.2015.i32.03 
Cuadrado Roura, J.R. (2010): "Las políticas de desarrollo regional y el conflicto eficiencia-equidad". Seminario Internacional de Desarrollo Económico Territorial, CEPAL. Santiago de Chile, 19-21 de octubre de 2010.

Champernowne, D.G. and Cowell, F.A. (1998): Economic inequality and income distribution. Cambridge University Press, Cambridge, 425p.

Daher, A. (2013): "El sector inmobiliario y las crisis económicas", Eure 39, 47-76. http://dx.doi.org/10.4067/S0250-71612013000300003

Foray, D.; David, P.A. and Hall, B.H. (2011): "Smart specialization. From academic idea to political instrument, the surprising career of a concept and the difficulties involved in its implementation", MTEI-working paper, Lausanne.

Fundación FOESSA y Cáritas (2012): Exclusión y Desarrollo Social. Fundación FOESSA y Cáritas Española, Madrid, 764p.

Fundación 1을 de Mayo y Fundación Francisco Largo Caballero (2011): El cambio de modelo productivo que España requiere para salir de la crisis. Fundación 10 de Mayo y Fundación Francisco Largo Caballero. [En línea] <http://www.1mayo.ccoo.es/nova/files/1018/ModeloProductivo.pdf>[15 de mayo de 2014].

Galor, O. (2000): "Income distribution and the process of development", European Economic Review 44, 706-712. http://dx.doi.org/10.1016/S0014-2921(99)00039-2

Hausmann, R. and Roderik, D. (2003): "Economic development as self-discovery", Journal of Development Economics 72, 603-633.

http://dx.doi.org/10.1016/S0304-3878(03)00124-X

Jordá, R. (2010): "Medio innovador y desarrollo local. Cambios en los medios innovadores. Evolución hacia la economía del conocimiento", in Rodríguez, F. (Coord.). Desarrollo local en tiempos de crisis ¿el retorno a los recursos endógenos?, pp. 113132, Universidad de Granada, AGE, IDR y Grupo de Desarrollo Local, Granada.

Jordá, R.; Ruiz, F.; González, R. and Contreras, G. (2014): “Caracterización del Sistema Nacional de Innovación español (SNI) y de los SRIs durante la crisis", in Albertos J. M. and Sánchez, J. L. (Coords.) Geografía de la crisis económica en España, pp. 263-304. Universidad de Valencia, Valencia.

Kitson, M.; Martin, R. and Tyler, P. (2011): "The geographies of austerity", Cambridge Journal of Regions, Economy and Society 4, 289-302.

http://dx.doi.org/10.1093/cjres/rsr030

ISSN: 0212-8594 ISSN-e: 2340-2776.№ DOI: http://dx.doi.org/10.12795/rea.2015.i32.03 
McCann, P. and Ortega-Argilés, R. (2013): "Smart specialisation, regional growth and applications to EU Cohesion Policy", Regional Studies (forthcoming, DOI: 10.1080/00343404.2013.799769)

Méndez, R. (2013): “Economía del conocimiento y nuevos contrastes territoriales en España: una perspectiva multiescalar", Boletín de la Asociación de Geógrafos Españoles 63, 7-32.

Méndez. R. (2014): "Crisis económica y reconfiguraciones territoriales", in Albertos J. M. and Sánchez, J. L. (Coords.) Geografía de la crisis económica en España, pp. 17-38. Universidad de Valencia, Valencia.

Méndez, R. and Sanchez Moral, S. (2011): "Spanish cities in the knowledge economy: theoretical debates and empirical evidences", European Urban and Regional Studies 18, 136-155. http://dx.doi.org/10.1177/0969776410381039

Ministerio de Trabajo e Inmigración (2010): El empleo y la dimensión social en la estrategia UE-2020. Ministerio de Trabajo e Inmigración, Madrid, 266p.

Musterd, S. and Murie, A. (2010): Making competitive cities. Pathways, actors and policies. Blackwell, London, 360p.

Naredo, J.M. (2009): "La cara oculta de la crisis. El fin del boom inmobiliario y sus consecuencias", Revista de Economía Crítica 7, 313-340.

Navarro Arancegui, M. and Gibaja Martins, J.J. (2009): "Las tipologías en los sistemas regionales de innovación. El caso de España”, Ekonomiaz 70, 241-281.

OCDE (2011a): Regions and Innovation Policy, OECD Reviews of Regional Innovation. OCDE, París, 325p.

OCDE (2011b): Divided We Stand: Why Inequality Keeps Rising. OCDE, París, 600p.

OIT (2012): Work Inequalities in the Crisis: Evidence from Europe. OIT, Ginebra, 600p.

Ondategui, J. C. (2008): Las tecnópolis en España. Thompson-Cívitas, Cizur-Menor (Navarra), 160p.

Ontiveros, E. (2011): "La desigualdad no es rentable", diario El País, Negocios, 1 de mayo de 2011 [En línea]

< http://elpais.com/diario/2011/05/01/negocio/1304255667_850215.html>[13 de mayo de 2014]. 
Ontiveros, E. (2012): "Perturbadora desigualdad", in Tezanos, J.F. Los nuevos problemas sociales. Duodécimo foro sobre tendencias sociales, pp. 19-36. Editorial Sistema, Madrid.

Pérez, F. (Dir.) (2011): Crecimiento y competitividad. Trayectoria y perspectivas de la economía española. Fundación BBVA-IVIE, Bilbao, 263p.

Piketty, T. (2014): Capital in the Twenty-First Century. Harvard University Press, Cambridge, 696p.

Pitarch, M.D. (2014): “Desigualdades regionales, pobreza y vulnerabilidad social en España durante la crisis (2007-2013)”, in Albertos J. M. and Sánchez, J. L. (Coords.) Geografía de la crisis económica en España, pp. 201-230. Universidad de Valencia, Valencia.

Puig Gómez, A. (2011): “El modelo productivo español en el periodo expansivo de 1997-2007: Insostenibilidad y ausencia de políticas de cambio", Revista de Economía Crítica 12, 65-81.

Rajan, R. (2010): "How inequality fueled the crisis", Proyect Syndicate, 9 de Julio de 2010 [En línea] <http://www.project-syndicate.org/commentary/how-inequalityfueled-the-crisis/spanish>[30 de mayo de 2014].

Rodríguez, E. and López, I. (2011): “Del auge al colapso. El modelo financieroinmobiliario de la economía española (1995-2010)", Revista de Economía Crítica 12, 39-63.

Rodríguez-Pose, A. and Tselios, V. (2010): "Inequalities in income and education and regional economic growth in western Europe", Annals of Regional Science 44, 349375. http://dx.doi.org/10.1007/s00168-008-0267-2

Romero, J.; Collado, J.C. and Rodríguez, M.T. (2014): "La economía política de la desigualdad. Los recortes en el sector público y sus repercusiones para el estado de bienestar en España", in Albertos J. M. and Sánchez, J. L. (Coords.) Geografía de la crisis económica en España, pp. 329-376. Universidad de Valencia, Valencia.

Salom, J. and Albertos, M.A. (2014): "La crisis económica en los distritos industriales valencianos", in Albertos J. M. and Sánchez, J. L. (Coords.) Geografía de la crisis económica en España, pp. 467-495. Universidad de Valencia, Valencia.

Sánchez, J. L. (2014): “Pautas regionales de las crisis económicas en España (19762012): ¿necesitamos un nuevo vocabulario?, in Albertos J. M. and Sánchez, J. L. (Coords.) Geografía de la crisis económica en España, pp. 173-200. Universidad de Valencia, Valencia.

ISSN: 0212-8594 ISSN-e: 2340-2776.N№ DOI: http://dx.doi.org/10.12795/rea.2015.i32.03 
Sánchez-Moral, S.; Méndez, R. and Arellano, A. (2014): "Creative economy and quality of employment in Large Urban Areas in Spain", Urban Geography 35, 264-289. http://dx.doi.org/10.1080/02723638.2013.876145

Spence, M. (2012): "Why do economies stop growing?", Proyect Syndicate, 23 de Julio de 2012 [En línea] <http://www.project-syndicate.org/commentary/why-doeconomies-stop-growing/spanish >[30 de mayo de 2014].

Stiglitz, J.E. (2012): El precio de la desigualdad. Taurus, Madrid, 498p.

\section{ANEXO I}

\begin{tabular}{|c|c|c|c|}
\hline \multicolumn{2}{|c|}{ Ramas de actividad } & \multirow{2}{*}{$\begin{array}{l}\text { Códigos CNAE93 rev.1 } \\
1,2\end{array}$} & \multirow[t]{2}{*}{ Códigos CNAE2009 } \\
\hline A & Agricultura, ganadería, caza... & & \\
\hline$B$ & Pesca & 5 & 3 \\
\hline $\mathrm{CA}$ & Extracción productos energéticos & 10,11 & 5,6 \\
\hline CB & Extrac. minerales no energéticos & 13,14 & $7,8,9$ \\
\hline DA & Alimentación, bebidas y tabaco & 15,16 & $10,11,12$ \\
\hline DB & Textil y de la confección & 17,18 & 13,14 \\
\hline DC & Cuero y del calzado & 19 & 15 \\
\hline DD & Madera y del corcho & 20 & 16 \\
\hline $\mathrm{DE}$ & Papel; edición, artes gráficas... & $21,22(f)$ & $17,18(f), 58(f)$ \\
\hline DF & Refino petróleo y comb. nucleares & 23 & 19 \\
\hline DG & Industria química & $24(a)$ & 20(a),21(a) \\
\hline $\mathrm{DH}$ & Caucho y materias plásticas & 25 & 22 \\
\hline DI & Otros prod. minerales no metálicos & 26 & 23 \\
\hline DJ & Metalurgia y productos metálicos & 27,28 & 24,25 \\
\hline DK & Maquinaria y equipo mecánico & 29 & 33,28 \\
\hline $\mathrm{DL}$ & Mat. eléctrico, electrónico y óptico & $30(a), 31,32(a), 33(a)$ & $26(a), 27$ \\
\hline DM & Material de transporte & $34,35(a)$ & $29,30(a)$ \\
\hline DN & Manufacturas diversas & 36,37 & $31,32,38$ \\
\hline E & Energía eléctrica, gas y agua & 40,41 & 35,36 \\
\hline $\mathrm{F}$ & Construcción & 45 & $41,42,43$ \\
\hline G & Comercio, reparación de vehículos... & $50,51,52$ & $45,46,47,95$ \\
\hline $\mathrm{H}$ & Hostelería & 55 & 55,56 \\
\hline 1 & Transporte, almac. y comunicaciones & $60,61,62,63,64(d)$ & $49,50,51,52,53(d), 61(d), 79$ \\
\hline j & Intermediación financiera & $65(b), 66(b), 67(b)$ & $64(b), 65(b), 66(b)$ \\
\hline K & $\begin{array}{l}\text { Act. inmobiliarias y serv. } \\
\text { empresariales }\end{array}$ & $70,71,72(\mathrm{c}), 73(\mathrm{c}), 74(\mathrm{c})$ & $\begin{array}{l}\text { 62(c),63(c),68,69(c),70(c),71(c), } \\
72(c), 73(c), 74(c), 77,78(c), 80(c), \\
81(c), 82(c)\end{array}$ \\
\hline $\mathrm{L}$ & Administración pública, defensa y SS & 75 & 84 \\
\hline M & Educación & $80(e)$ & 85(e) \\
\hline $\mathrm{N}$ & Act. sanitarias y veter.; serv. sociales & $85(e)$ & 75(e),86(e),87(e),88(e) \\
\hline
\end{tabular}

ISSN: 0212-8594 ISSN-e: 2340-2776.N№ DOI: http://dx.doi.org/10.12795/rea.2015.i32.03

REA 32 (2015): 49-75

http://editorial.us.es/es/revista-de-estudios-andaluces 
Simón Sánchez-Moral

Territorio, transformación productiva, equidad y redes.

\begin{tabular}{|l|l|l|l|}
\hline O & Otras actividades sociales & $90,91,92(\mathrm{f}), 93$ & $\begin{array}{l}37,39,59(\mathrm{f}), 60(\mathrm{f}), 90(\mathrm{f}), 91(\mathrm{f}), \\
92(\mathrm{f}), 93(\mathrm{f}), 94\end{array}$ \\
\hline P & Actividades de los hogares & 95 & 97 \\
\hline Q & Organismos extraterritoriales & 99 & 99 \\
\hline Composición clusters innovadores: & \\
(a) Industrias alta intensidad tecnológica & \\
(b) Actividades financieras \\
(c) Servicios a empresas intensivos en conocimiento \\
(d) Telecomunicaciones \\
(e) Educación, sanidad y servicios sociales \\
(f) Industrias culturales
\end{tabular}

Fuente: Elaboración propia.

ISSN: 0212-8594 ISSN-e: 2340-2776.№ DOI: http://dx.doi.org/10.12795/rea.2015.i32.03

REA 32 (2015): 49-75

http://editorial.us.es/es/revista-de-estudios-andaluces 